\title{
A DIRECT METHOD FOR COMPUTING HIGHER ORDER FOLDS*
}

\author{
ZHONG-HUA YANG $\dagger$ AND H. B. KELLER $\ddagger$
}

\begin{abstract}
We consider the computation of higher order fold or limit points of two parameter-dependent nonlinear problems. A direct method is proposed and an efficient implementation of the direct method is presented. Numerical results for the thermal ignition problem are given.
\end{abstract}

Key words. two-parameter nonlinear problems, simple limit points, higher order fold points, double extended systems, Newton's methods

1. Introduction. This paper is concerned with the computation of special kinds of singular points, which are called (simple) higher order fold or limit points. They may arise in two parameter nonlinear problems of the form

$$
f(\lambda, \mu, x)=0
$$

where $\lambda, \mu \in \mathbb{R}, x \in X$, a Banach space, and $f$ is a $C^{3}$ mapping from $\mathbb{R} \times \mathbb{R} \times X \rightarrow X$. A problem in the theory of thermal ignition is one such problem [1], [2], [3] which we treat. Two parameter nonlinear problems arise in many other physical applications [6], [8]. The problem in thermal ignition has the form

$$
\begin{aligned}
& L x=h(\lambda, \mu, x), \\
& B x=0
\end{aligned}
$$

where $L$ is a uniformly elliptic differential operator, $B$ is a boundary operator, $\lambda$ is a rate parameter, $\mu$ is related to the activation energy, and $h$ has the form

$$
h(\lambda, \mu, x)=\lambda \exp \left(\frac{x}{1+\mu x}\right) .
$$

The solution $x$ is the dimensionless temperature. Of particular interest are the values $\lambda_{0}$ and $\mu_{0}$ which correspond to the loss of criticality in the exothermic reaction described by (1.2). These values correspond to "folds" or "limit" points.

Spence and Werner [10] proved that a cubic fold point $\left(\lambda_{0}, \mu_{0}, x_{0}\right)$ of $f$ with regard to $\lambda$ corresponds to a quadratic fold point $\left(\lambda_{0}, \mu_{0}, x_{0}, \phi_{0}\right)$ of an extended system, $F$, of $f$, provided certain conditions are satisfied. They located the cubic fold by using a continuation method [5] to compute the quadratic fold point of an "extended system". The main idea in this paper is to reduce a problem with cubic folds to a regular problem by using a larger "double extended system". We also present an efficient implementation for solving the larger "double extended system". Thus we locate a cubic fold directly, without any continuation. Related techniques in [11] show how to find isolas and cusps using extended systems.

In $\S 2$ we give a brief review of simple fold points, the degree of a fold, and extended systems. The main idea of our treatment of higher degree fold points is contained in Theorem 2.1. The efficient implementation of Newton's method is given in $\S 3$. In $\S 4$ we give numerical results.

* Received by the editors May 15, 1984, and in revised form February 25, 1985. This work was supported by the U.S. Department of Energy under contract EY-76-S-03-0767, project agreement no. 12, and the U.S. Army Research Office under contract DAAG 29-78-C-0011.

$\dagger$ Department of Mathematics, Shanghai University of Science and Technology, The People's Republic of China.

$\ddagger$ Applied Mathematics, California Institute of Technology, Pasadena, California 91125 . 
2. Folds, degree of a fold, and extended systems. First we review some of the definitions and the main results about folds. Let $Y$ be a Banach space and consider the $C^{3}$ mapping

$$
F:\left\{\begin{array}{l}
\mathbb{R} \times Y \rightarrow Y, \\
(\mu, y) \rightarrow F(\mu, y) .
\end{array}\right.
$$

We use the notation $F_{\mu}(a), F_{\mu \mu}(a), F_{y}(a), F_{\mu y}(a), F_{y y}(a), F_{y y y}(a), \cdots$, to denote the partial Fréchet-derivatives of $F$ at $a=(\mu, y) \in \mathbb{R} \times Y$. We denote the dual pairing of $y \in Y$ and $\psi \in Y^{*}$ by $\psi y$.

Definition 2.1. A point $a_{0}=\left(\mu_{0}, y_{0}\right) \in \mathbb{R} \times Y$ is a fold point of $F$ (with respect to $\mu)$ if

$$
\begin{aligned}
& F\left(a_{0}\right)=0, \\
& \text { Ker } F_{y}\left(a_{0}\right) \neq 0, \\
& F_{\mu}\left(a_{0}\right) \notin \text { Range } F_{y}\left(a_{0}\right) .
\end{aligned}
$$

Definition 2.4. A fold point $a_{0}$ is a simple fold of $F$ if in addition to (2.1)-(2.3)

$$
\operatorname{dim} \operatorname{Ker} F_{y}\left(a_{0}\right)=\text { codim Range } F_{y}\left(a_{0}\right)=1 \text {. }
$$

In this case there exist nontrivial $\phi_{0} \in Y$ and $\psi_{0} \in Y^{*}$ such that

$$
\begin{aligned}
& \operatorname{Ker} F_{y}\left(a_{0}\right)=\left\{\alpha \phi_{0} \mid \alpha \in \mathbb{R}\right\}, \\
& \text { Range } F_{y}\left(a_{0}\right)=\left\{y \in Y \mid \psi_{0} y=0\right\} .
\end{aligned}
$$

As is well known, near a simple fold point $a_{0}$, the zero set of $F$, denoted by $F^{-1}(0)$, is a smooth curve

$$
\Gamma: F^{-1}(0) \cap \cup=\left\{[\mu(s), y(s)]|| s-s_{0} \mid \leqq \delta\right\} .
$$

Here $U$ is a neighborhood of the fold point $a_{0}, \delta$ is positive and $\mu(\cdot), y(\cdot)$ are smooth mappings satisfying

$$
\mu\left(s_{0}\right)=\mu_{0}, \quad y\left(s_{0}\right)=y_{0}, \quad\left|\mu^{\prime}(s)\right|+\left\|y^{\prime}(s)\right\|>0 .
$$

Along $\Gamma$ we have the identity

$$
F(\mu(s), y(s))=0
$$

and we can differentiate it with respect to $s$ as many times as the smoothness of $F$ allows. In place of $F_{\mu}(\mu(s), y(s)), \cdots, F_{y y y}(\mu(s), y(s))$, we shall write $F_{\mu}(s), \cdots, F_{y y y}(s)$. Then we get by differentiating in $(2.5 \mathrm{a})$

$$
F_{\mu}(s) \mu^{\prime}(s)+F_{y}(s) y^{\prime}(s) \equiv 0, \quad\left|s-s_{0}\right|<\delta .
$$

Obviously (2.3) and (2.4) imply from (2.5) evaluated at $s_{0}$, that

$$
\begin{aligned}
& \mu^{\prime}\left(s_{0}\right)=0, \\
& y^{\prime}\left(s_{0}\right)=\alpha \phi_{0} \quad \text { for some } \alpha \in \mathbb{R}, \alpha \neq 0 \text { (say } \alpha=1 \text { ). }
\end{aligned}
$$

The first nonvanishing derivative of $\mu(s)$ at $s_{0}$ determines the "degree" of the fold. We formalize this in

Definition 2.7. A simple fold point $a_{0} \in \mathbb{R} \times Y$ is said to have degree $m$ if $d^{p} \mu\left(s_{0}\right) / d s^{p}=0$ for all $p<m$ and $d^{m} \mu\left(s_{0}\right) / d s^{m} \neq 0$.

The result in (2.6) implies that all simple folds have degree two or greater. To actually find the degree of a simple fold we need only differentiate further in (2.5a) 
or $(2.5 \mathrm{~b})$ and find the first nonvanishing derivative of $\mu(s)$ at $s_{0}=s$. Thus from (2.5b) we obtain

$$
\begin{aligned}
F_{\mu}(s) \mu^{\prime \prime}(s) & +F_{y}(s) y^{\prime \prime}(s)+F_{\mu \mu}(s) \mu^{\prime}(s) \mu^{\prime}(s)+2 F_{\mu y}(s) \mu^{\prime}(s) y^{\prime}(s) \\
& +F_{y y}(s) y^{\prime}(s) y^{\prime}(s)=0, \quad\left|s-s_{0}\right|<\delta .
\end{aligned}
$$

With a simple fold at $s=s_{0}$ we use (2.6) and (2.4) in the above, apply $\psi_{0}$ and note that $(2.3)$ and $(2.4 \mathrm{c})$ imply $\psi_{0} F_{\mu}\left(s_{0}\right) \neq 0$ to get

$$
\mu^{\prime \prime}\left(s_{0}\right)=\frac{\psi_{0} F_{y y}\left(s_{0}\right) \phi_{0} \phi_{0}}{\psi_{0} F_{\mu}\left(s_{0}\right)} .
$$

So a simple fold is of degree two if and only if

$$
\psi_{0} F_{y y}\left(s_{0}\right) \phi_{0} \phi_{0} \neq 0
$$

We introduce the extended or inflated mapping

$$
G:\left\{\begin{array}{l}
\mathbb{R} \times Y \times Y \rightarrow \mathbb{R}+Y \times Y, \\
(\mu, y, \phi) \rightarrow\left(\begin{array}{c}
l \phi-1 \\
F(\mu, y) \\
F_{y}(\mu, y) \phi
\end{array}\right),
\end{array}\right.
$$

where $l \in Y^{*}$ is chosen later on in $\S 3$. It is not difficult to show (see [10, Thm. 2.1]) that if

$$
G\left(\mu_{0}, y_{0}, \phi_{0}\right)=0
$$

and $\left(\mu_{0}, y_{0}\right)$ is a simple fold of $F$ of degree two, then

$$
D G^{0}=\left[\begin{array}{ccc}
0 & 0 & l \\
F_{\mu}^{0} & F_{y}^{0} & 0 \\
F_{\mu y}^{0} \phi_{0} & F_{y y}^{0} \phi_{0} & F_{y}^{0}
\end{array}\right]
$$

is nonsingular. As a consequence, the system $G(\mu, y, \phi)=0$ can be solved by Newton's method in some neighborhood of $\left(\mu_{0}, y_{0}, \phi_{0}\right) . G(\mu, y, \phi)=0$ is called an extended system for $F(\mu, y)=0$. Various kinds of extended systems have been used by different authors [6], [9], [10] following their introduction by Keener and Keller in [4].

We next consider two parameter nonlinear problems involving the smooth mapping

$$
f:\left\{\begin{array}{l}
\mathbb{R} \times \mathbb{R} \times X \rightarrow X, \\
(\lambda, \mu, x) \rightarrow f(\lambda, \mu, x) .
\end{array}\right.
$$

For some fixed value of $\mu=\mu_{0}$ we assume that

$$
g(\lambda, x) \equiv f\left(\lambda, \mu_{0}, x\right)=0
$$

has a simple fold point $\left(\lambda_{0}, x_{0}\right)$ with respect to $\lambda$, according to Definitions 2.1 and 2.4.

We introduce, in exact analogy with (2.8), an extended system for $f(\lambda, \mu, x)=0$

$$
F(\lambda, \mu, x, \phi) \equiv\left(\begin{array}{c}
l \phi-1 \\
f(\lambda, \mu, x) \\
f_{x}(\lambda, \mu, x) \phi
\end{array}\right)=0 .
$$

Here $F$ is a mapping from $\mathbb{R} \times \mathbb{R} \times X \times X$ to $\mathbb{R} \times X \times X$. If we denote $Y \equiv \mathbb{R} \times X \times X$ and $y \equiv(\lambda, x, \phi)^{T} \in Y$, the extended system $F(\lambda, \mu, x, \phi)=0$ can be written as $F(\mu, y)=0$. 
Our main idea is to extend this extended system, $F(\mu, \lambda)=0$, again and to get the doubly extended system

$$
H(\mu, y, \Phi) \equiv\left(\begin{array}{c}
L \Phi-1 \\
F(\mu, y) \\
F_{y}(\mu, y) \Phi
\end{array}\right)=0
$$

where $\Phi \in Y, L \in Y^{*}$. A specific $L \equiv(0, l, 0)$ will be chosen later on in order to simply (2.12). Using this system we obtain

Theorem 2.13. Assume $F_{\mu}^{0} \notin$ Range $F_{y}^{0}$. Then a third degree simple fold point $\left(\lambda_{0}, \mu_{0}, x_{0}\right)$ of $f(\lambda, \mu, x)$ with respect to $\lambda$ corresponds to a regular solution $\left(\lambda_{0}, \mu_{0}, x_{0}, \phi_{0}, v_{0}\right)$ of the inflated system

$$
\left(\begin{array}{c}
l \phi-1 \\
l v \\
f(\lambda, \mu, x) \\
f_{x}(\lambda, \mu, x) \phi \\
f_{x x} \phi \phi+f_{x} v
\end{array}\right)=0
$$

Proof. According to Spence and Werner [10, Thm. 3.1] a third degree fold point $\left(\lambda_{0}, \mu_{0}, x_{0}\right)$ of $f(\lambda, \mu, x)$ with respect to $\lambda$ corresponds to a second degree fold point $\left(\mu_{0}, y_{0}\right)=\left(\lambda_{0}, \mu_{0}, x_{0}, \phi_{0}\right)$ of $F(\mu, y)$ in $(2.11)$ with respect to $\mu$ provided $F_{\mu}^{0} \notin$ Range $F_{y}^{0}$. Further applying [10, Thm. 2.1] to $F(\mu, y)$ we get that a second degree fold point $\left(\mu_{0}, y_{0}\right)$ of $F(\mu, y)$ with respect to $\mu$ corresponds to a regular solution $\left(\mu_{0}, y_{0}, \Phi_{0}\right)$ of the doubly extended system $H(\mu, y, \Phi)=0$ in (2.12) i.e. $H(\mu, y, \Phi)=0$ is a regular system at $\left(\mu_{0}, y_{0}, \Phi_{0}\right)$, provided $L \Phi=1$.

Next we show that the double extended system $H(\mu, y, \Phi)=0$ is equivalent to (2.14) for a particular $L$. Let

$$
\Phi=\left(\begin{array}{c}
\sigma \\
u \\
v
\end{array}\right)
$$

and choose $L=(0, l, 0)$. Then (2.12) becomes

$$
\begin{aligned}
& L \Phi-1=l u-1=0, \\
& F(\mu, y)=\left(\begin{array}{c}
l \phi-1 \\
f(\lambda, \mu, x) \\
f_{x}(\lambda, \mu, x) \phi
\end{array}\right)=0 \\
& F_{y}(\mu, y) \Phi=\left(\begin{array}{ccc}
0 & 0 & l \\
f_{\lambda} & f_{x} & 0 \\
f_{\lambda x} \phi & f_{x x} \phi & f_{x}
\end{array}\right)\left(\begin{array}{l}
\sigma \\
u \\
v
\end{array}\right)=\left(\begin{array}{c}
l v \\
\sigma f_{\lambda}+f_{x} u \\
\sigma f_{\lambda x} \phi+f_{x x} \phi u+f_{x} v
\end{array}\right)=0 .
\end{aligned}
$$

By Definition 2.1, we know that $f_{\lambda} \notin$ Range $f_{x}$ at a fold point. From (2.15f): $\sigma f_{\lambda}+f_{x} u=0$. We thus get $\sigma=0$ and then $u \in N\left(f_{x}\right)$. From (2.15d) and Definition 2.4 of a simple fold we have $u=\alpha \phi_{0}$. Using this $u$ in (2.15a) we get $\alpha=1$ in order to satisfy (2.15b). The solution of $(2.15)$ is thus

$$
\mu=\mu_{0}, \quad y=y_{0} \equiv\left(\begin{array}{c}
\lambda_{0} \\
x_{0} \\
\phi_{0}
\end{array}\right), \quad \Phi=\Phi_{0} \equiv\left(\begin{array}{c}
0 \\
\phi_{0} \\
v_{0}
\end{array}\right) .
$$


Here $v_{0}$ satisfies

$$
l v_{0}=0, \quad f_{x x}^{0} \phi_{0} \phi_{0}+f_{x}^{0} v_{0}=0,
$$

and $\mu_{0}$ and $y_{0}$ satisfy $F\left(\mu_{0}, y_{0}\right)=0$. This shows that $\left(\lambda_{0}, \mu_{0}, x_{0}, \phi_{0}, v_{0}\right)$ is also a solution of (2.14).

On the other hand, if we know the solution $\left(\lambda_{0}, \mu_{0}, x_{0}, \phi_{0}, v_{0}\right)$ of $(2.14)$, we can easily construct a solution of (2.15) as in (2.16). Actually we have reduced (2.15) to (2.14), which is also a regular system, by choosing the particular $L \equiv(0, l, 0)$.

Since the inflated system (2.14) is regular, we can solve it by using Newton's method. The solution of (2.14) is just the third degree fold point with respect to $\lambda$ of the original two parameter nonlinear problem, $f(\lambda, \mu, x)=0$.

We now turn to the efficient solutions of (2.14).

3. Efficient implementation of Newton's method. After discretization (2.14) becomes a finite-dimensional nonlinear system. Let $x, \phi, v \in E^{n}$, the dimension of (2.14) is actually $3 n-2$ because we can choose $l \phi=\phi_{r}=1, l v=v_{r}=0$, where $r$ is a positive integer in $1 \leqq r \leqq n$. For convenience we shall choose $r=1$ and the discretized system of (2.14) is denoted by the same notation. Newton's method applied to (2.14) yields:

$$
\left[\begin{array}{ccccc}
0 & 0 & 0 & l & 0 \\
0 & 0 & 0 & 0 & l \\
f_{\mu} & f_{\lambda} & f_{x} & 0 & 0 \\
f_{\mu x} \phi & f_{\lambda x} \phi & f_{x x} \phi & f_{x} & 0 \\
f_{\mu x x} \phi \phi+f_{\mu x} v & f_{\lambda x x} \phi \phi+f_{\lambda x} v & f_{x x x} \phi \phi+f_{x x} v & 2 f_{x x} \phi & f_{x}
\end{array}\right]^{(\nu)}\left[\begin{array}{c}
\mu^{\nu+1}-\mu^{\nu} \\
\lambda^{\nu+1}-\lambda^{\nu} \\
x^{\nu+1}-x^{\nu} \\
\phi^{\nu+1}-\phi^{\nu} \\
v^{\nu+1}-v^{\nu}
\end{array}\right]
$$

$$
=\left[\begin{array}{c}
-l \phi+1 \\
-l v \\
-f \\
-f_{x} \phi \\
-f_{x x} \phi \phi-f_{x} v
\end{array}\right]^{(\nu)}
$$

Here superscript $(\nu)$ denotes evaluation of the coefficient matrix and the right-hand side at $\left(\mu^{\nu}, \lambda^{\nu}, x^{\nu}, \phi^{\nu}, v^{\nu}\right)$. The starting value is $\left(\mu^{0}, \lambda^{0}, x^{0}, \phi^{0}, v^{0}\right)$. We write $\delta \mu^{\nu}=$ $\mu^{\nu+1}-\mu^{\nu}, \delta \lambda^{\nu}=\lambda^{\nu+1}-\lambda^{\nu}, \delta x^{\nu}=x^{\nu+1}-x^{\nu}, \delta \phi^{\nu}=\phi^{\nu+1}-\phi^{\nu}, \delta v^{\nu}=v^{\nu+1}-v^{\nu}$.

In expanded form, and with the superscripts of $(\delta \mu, \delta \lambda, \delta x, \delta \phi, \delta v)$ suppressed, (3.1) can be written as

$$
\begin{aligned}
& \delta \phi_{1}=0, \\
& \delta \nu_{1}=0, \\
& \mathbb{A} \delta x+\delta \lambda \cdot \mathbb{D}_{1}+\delta \mu \cdot \mathbb{D}_{2}=\mathbb{C}_{1}, \\
& \mathbb{A} \delta \phi+\delta \lambda \cdot \mathbb{D}_{3}+\delta \mu \cdot \mathbb{D}_{4}+\mathbb{B}_{1} \delta x=\mathbb{C}_{2}, \\
& \mathbb{A} \delta v+\delta \lambda \cdot \mathbb{D}_{5}+\delta \mu \cdot \mathbb{D}_{6}+2 \mathbb{B}_{1} \delta \phi+\mathbb{B}_{2} \delta x=\mathbb{C}_{3} .
\end{aligned}
$$

Here we have introduced

$$
\begin{aligned}
& \mathbb{A}=f_{x}\left(\lambda^{\nu}, \mu^{\nu}, x^{\nu}\right), \quad \mathbb{B}_{1}=f_{x x}\left(\lambda^{\nu}, \mu^{\nu}, x^{\nu}\right) \phi^{\nu}, \\
& \mathbb{B}_{2}=f_{x x x}\left(\lambda^{\nu}, \mu^{\nu}, x^{\nu}\right) \phi^{\nu} \phi^{\nu}+f_{x x}\left(\lambda^{\nu}, \mu^{\nu}, x^{\nu}\right) v^{\nu},
\end{aligned}
$$




$$
\begin{aligned}
& \mathbb{D}_{1}=f_{\lambda}\left(\lambda^{\nu}, \mu^{\nu}, x^{\nu}\right), \quad \mathbb{D}_{2}=f_{\mu}\left(\lambda^{\nu}, \mu^{\nu}, x^{\nu}\right), \\
& \mathbb{D}_{3}=f_{\lambda x}\left(\lambda^{\nu}, \mu^{\nu}, x^{\nu}\right) \phi^{\nu}, \quad \mathbb{D}_{4}=f_{\mu x}\left(\lambda^{\nu}, \mu^{\nu}, x^{\nu}\right) \phi^{\nu}, \\
& \mathbb{D}_{5}=f_{\lambda x x}\left(\lambda^{\nu}, \mu^{\nu}, x^{\nu}\right) \phi^{\nu} \phi^{\nu}+f_{\lambda x}\left(\lambda^{\nu}, \mu^{\nu}, x^{\nu}\right) v^{\nu}, \\
& \mathbb{D}_{6}=f_{\mu x x}\left(\lambda^{\nu}, \mu^{\nu}, x^{\nu}\right) \phi^{\phi} \gamma^{\nu}+f_{\mu x}\left(\lambda^{\nu}, \mu^{\nu}, x^{\nu}\right) v^{\nu}, \\
& \mathbb{C}_{1}=-f\left(\lambda^{\nu}, \mu^{\nu}, x^{\nu}\right), \quad \mathbb{C}_{2}=-f_{x}\left(\lambda^{\nu}, \mu^{\nu}, x^{\nu}\right) \phi^{\nu}, \\
& \mathbb{C}_{3}=-f_{x x}\left(\lambda^{\nu}, \mu^{\nu}, x^{\nu}\right) \phi^{\nu} \phi^{\nu}-f_{x}\left(\lambda^{\nu}, \mu^{\nu}, x^{\nu}\right) v^{\nu} .
\end{aligned}
$$

Nowlet

$$
\begin{aligned}
& \delta s^{T}=\left(\delta \lambda, \delta x_{2}-\delta x_{1} \phi_{2}^{\nu}, \cdots, \delta x_{n}-\delta x_{1} \phi_{n}^{\nu}\right), \\
& \delta t^{T}=\left(\delta \lambda, \delta \phi_{2}, \cdots, \delta \phi_{n}\right), \\
& \delta r^{T}=\left(\delta \lambda, \delta v_{2}, \cdots, \delta v_{n}\right),
\end{aligned}
$$

and

$$
\mathscr{A}=\left(\mathbb{D}_{1}: \tilde{A}\right),
$$

i.e., $\mathbb{A}$ with first column replaced by $\mathbb{D}_{1}$. We rewrite $(3.4),(3.5),(3.6)$ as

$$
\begin{aligned}
& \mathscr{A} \delta s=\mathbb{C}_{1}+\delta x_{1} \cdot \mathbb{C}_{2}-\delta \mu \cdot \mathbb{D}_{2}, \\
& \mathscr{A} \delta t=\mathbb{C}_{2}-\mathbb{B}_{1} \delta x+\delta \lambda \cdot\left(\mathbb{D}_{1}-\mathbb{D}_{3}\right)-\delta \mu \cdot \mathbb{D}_{4}, \\
& \mathscr{A} \delta r=\mathbb{C}_{3}-2 \mathbb{B}_{1} \delta \phi-\mathbb{B}_{2} \delta x+\delta \lambda \cdot\left(\mathbb{D}_{1}-\mathbb{D}_{5}\right)-\delta \mu \cdot \mathbb{D}_{6} .
\end{aligned}
$$

Close to the fold point, $\mathscr{A}$ will be nonsingular by [7, Thm. 1] with $P x=x-x_{1} \phi^{\nu}$ and the condition $(I-P) \phi_{0} \neq 0$ is satisfied by the $\phi_{0}$ given in $(2.4 \mathrm{~b})$. Thus (3.8) can be solved for $\delta s$ in terms of $\delta x_{1}$ and $\delta \mu$. By solving $\mathscr{A} \alpha=\mathbb{C}_{1}, \mathscr{A} \beta=\mathbb{C}_{2}, \mathscr{A} \xi=\mathbb{D}_{2}$ we obtain

$$
\delta s=\alpha+\delta x_{1} \cdot \beta-\delta \mu \cdot \xi
$$

i.e.,

$$
\begin{aligned}
& \delta \lambda=\alpha_{1}+\delta x_{1} \cdot \beta_{1}-\delta \mu \cdot \xi_{1}, \\
& \delta x^{T}=\left(\delta x_{1}, \alpha_{2}+\delta x_{1}\left(\beta_{2}+\phi_{2}^{\nu}\right)-\delta \mu \cdot \xi_{2}, \cdots, \alpha_{n}+\delta x_{1}\left(\beta_{n}+\phi_{n}^{\nu}\right)-\delta \mu \cdot \xi_{n}\right) .
\end{aligned}
$$

Substituting (3.11), (3.12) into (3.9) gives

$$
\mathscr{A} \delta t=\mathbb{C}_{4}+\delta x_{1} \cdot \mathbb{C}_{5}+\delta \mu \cdot \mathbb{C}_{6},
$$

where

$$
\begin{aligned}
& \mathbb{C}_{4}=\mathbb{C}_{2}-\mathbb{B}_{1}\left(\begin{array}{c}
0 \\
\alpha_{2} \\
\vdots \\
\alpha_{n}
\end{array}\right)+\alpha_{1}\left(\mathbb{D}_{1}-\mathbb{D}_{3}\right), \\
& \mathbb{C}_{5}=-\mathbb{B}_{1}\left(\begin{array}{c}
1 \\
\beta_{2}+\phi_{2}^{\nu} \\
\vdots \\
\beta_{n}+\phi_{n}^{\nu}
\end{array}\right)+\beta_{1}\left(\mathbb{D}_{1}-\mathbb{D}_{3}\right),
\end{aligned}
$$




$$
\mathbb{C}_{6}=\mathbb{B}_{1}\left(\begin{array}{c}
0 \\
\xi_{2} \\
\vdots \\
\xi_{n}
\end{array}\right)-\xi_{1}\left(\mathbb{D}_{1}-\mathbb{D}_{3}\right)-\mathbb{D}_{4} .
$$

Then (3.9) can be solved for $\delta t$ in terms of $\delta x_{1}$ and $\delta \mu$. By solving $\mathscr{A} \gamma=\mathbb{C}_{4}, \mathscr{A} \eta=\mathbb{C}_{5}$, $\mathscr{A} \zeta=\mathbb{C}_{6}$ we get

i.e.,

$$
\delta t=\gamma+\delta x_{1} \cdot \eta+\delta \mu \cdot \zeta
$$

$$
\begin{aligned}
& \delta \lambda=\gamma_{1}+\delta x_{1} \cdot \eta_{1}+\delta \mu \cdot \zeta_{1}, \\
& \delta \phi^{T}=\left(0, \gamma_{2}+\delta x_{1} \cdot \eta_{2}+\delta \mu \cdot \zeta_{2}, \cdots, \gamma_{n}+\delta x_{1} \cdot \eta_{n}+\delta \mu \cdot \zeta_{n}\right) .
\end{aligned}
$$

Substituting (3.11), (3.12), (3.14) into (3.10) gives

$$
\mathscr{A} \delta r=\mathbb{C}_{7}+\delta x_{1} \mathbb{C}_{8}+\delta \mu \mathbb{C}_{9},
$$

where

$$
\begin{aligned}
& \mathbb{C}_{7}+\mathbb{C}_{3}-2 \mathbb{B}_{1}\left(\begin{array}{c}
0 \\
\gamma_{2} \\
\vdots \\
\gamma_{n}
\end{array}\right)-\mathbb{B}_{2}\left(\begin{array}{c}
0 \\
\alpha_{2} \\
\vdots \\
\alpha_{n}
\end{array}\right)+\alpha_{1}\left(\mathbb{D}_{1}-\mathbb{D}_{7}\right), \\
& \mathbb{C}_{8}=-2 \mathbb{B}_{1}\left(\begin{array}{c}
0 \\
\eta_{2} \\
\vdots \\
\eta_{n}
\end{array}\right)-\mathbb{B}_{2}\left(\begin{array}{c}
1 \\
\beta_{2}+\phi_{2}^{\nu} \\
\vdots \\
\beta_{n}+\phi_{n}^{\nu}
\end{array}\right)+\beta_{1}\left(\mathbb{D}_{1}-\mathbb{D}_{5}\right), \\
& \mathbb{C}_{9}=-2 \mathbb{B}_{1}\left(\begin{array}{c}
0 \\
\zeta_{2} \\
\vdots \\
\zeta_{n}
\end{array}\right)+\mathbb{B}_{2}\left(\begin{array}{c}
0 \\
\xi_{2} \\
\vdots \\
\xi_{n}
\end{array}\right)-\mathbb{D}_{6}-\xi_{1}\left(\mathbb{D}_{1}-\mathbb{D}_{5}\right) .
\end{aligned}
$$

Now (3.10) can be solved for $\delta r$ in terms of $\delta x_{1}$ and $\delta \mu$. By solving $\mathscr{A} \varepsilon=\mathbb{C}_{7}, \mathscr{A} \sigma=\mathbb{C}_{8}$, $A \zeta=\mathbb{C}_{9}$ we get:

$$
\delta r=\varepsilon+\delta x_{1} \cdot \sigma+\delta \mu \cdot \tau
$$

Thus

$$
\begin{aligned}
& \delta \lambda=\varepsilon_{1}+\delta x_{1} \cdot \sigma_{1}+\delta \mu \cdot \tau_{1}, \\
& \delta v^{T}=\left(0, \varepsilon_{2}+\delta x_{2} \cdot \sigma_{2}+\delta \mu \cdot \tau_{2}, \cdots, \varepsilon_{n}+\delta x_{1} \cdot \sigma_{n}+\delta \mu \cdot \tau_{n}\right) .
\end{aligned}
$$

Finally we solve for $\delta \lambda, \delta \mu$ and $\delta x_{1}$ from (3.11), (3.13), (3.15) and we get $\delta x, \delta \phi, \delta v$ by substituting $\delta \lambda, \delta \mu$ and $\delta x_{1}$ into (3.12), (3.14), (3.16). This concludes one step of Newton's method (3.1) applied to (2.14). Our indicated algorithm for solving the linear system defining the Newton iterates is similar to one proposed in [7].

4. Numerical example. We consider the boundary value problem

$$
\begin{aligned}
& f(\lambda, \mu, x) \equiv x^{\prime \prime}+\lambda \exp \left(\frac{x}{1+\mu x}\right)=0, \\
& x(0)=x(1)=0
\end{aligned}
$$



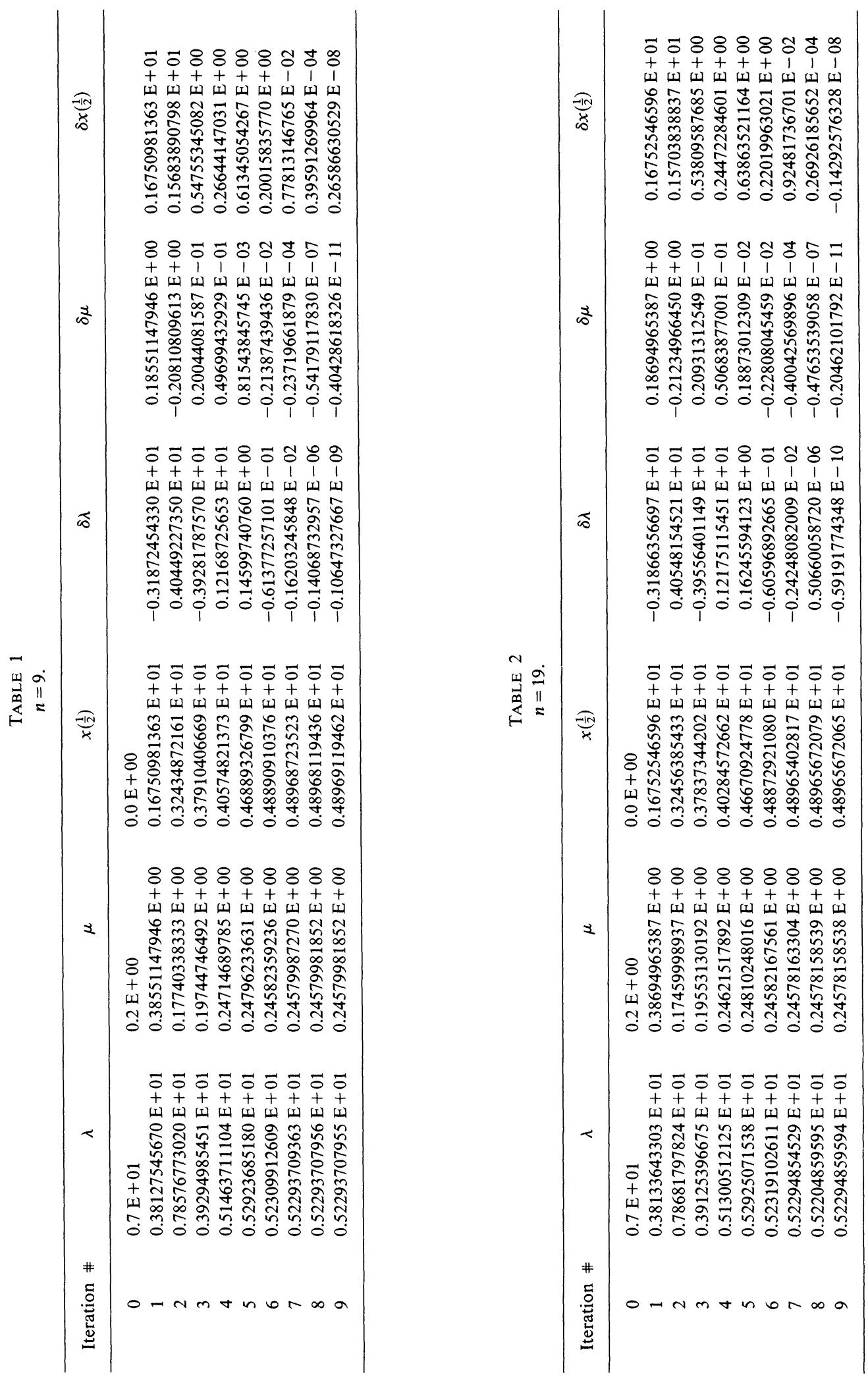


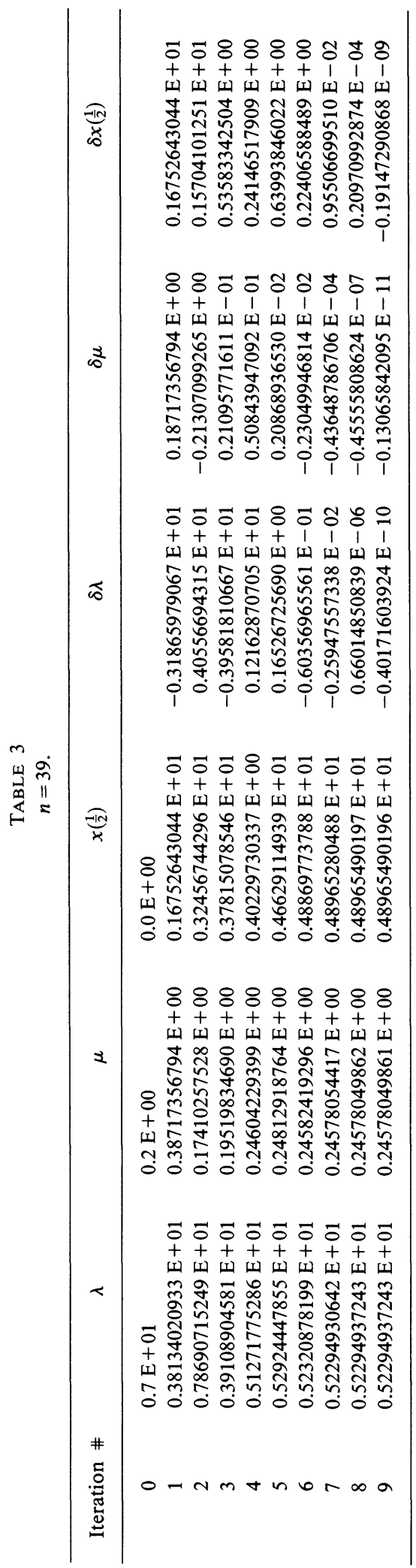


which describes an exothermic chemical reaction in an infinite slab [3]. It is discretized on the mesh $t_{j}=j h, j=0,1,2, \cdots, n+1$ using the Collatz Mehrstellenverfahren:

$$
x\left(t_{j-1}\right)-2 x\left(t_{j}\right)+x\left(t_{j+1}\right)-\frac{h^{2}}{12}\left[x^{\prime \prime}\left(t_{j-1}\right)+10 x^{\prime \prime}\left(t_{j}\right)+x^{\prime \prime}\left(t_{j+1}\right)\right]=h^{2} x^{\prime \prime}\left(t_{j}\right)+O\left(h^{6}\right) .
$$

The discretized form of (4.1) is thus:

$$
A x+E(\lambda, \mu, x)=0, x_{0}=x_{n+1}=0, \quad x \in \mathbb{R}^{n},
$$

where

$$
\begin{aligned}
& E \equiv\left(E_{1}, \cdots, E_{n}\right)^{T}, \\
& E_{i} \equiv \frac{h^{2}}{12} \cdot \lambda\left[\exp \left(\frac{x_{i-1}}{1+\mu x_{i-1}}\right)+10 \exp \left(\frac{x_{i}}{1+\mu x_{i}}\right)+\exp \left(\frac{x_{i+1}}{1+\mu x_{i+1}}\right)\right]
\end{aligned}
$$

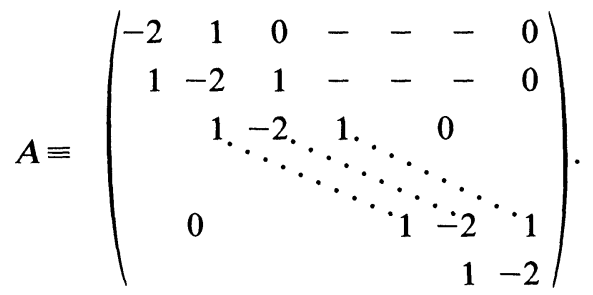

The double extended system now has the form

$$
\left\{\begin{array}{c}
l \phi-1 \\
A x+E(\lambda, \mu, x) \\
{\left[A+E_{x}(\lambda, \mu, x)\right] \phi} \\
E_{x x}(\lambda, \mu, x) \phi \phi+\left[A+E_{x}(\lambda, \mu, x)\right] v \\
l v
\end{array}\right\}=0 .
$$

We choose $l$ so that $l \phi=\phi_{m}, l v=v_{m}$, where $m=(n+1) / 2$. (Of course we must choose $n$ odd.) The calculation of each Newton's step requires solving nine $n \times n$ systems with the same coefficient matrix. The results of computation are given in tables 1, 2 and 3. They show good agreement with the results in [10].

\section{REFERENCES}

[1] N. W. BAZLEY AND G. C. WAKE, The disappearance of criticality in the theory of thermal ignition, Z. Angew. Math. Phys., 29 (1979), pp. 971-976.

[2] T. Boddington, P. GRAy AND C. Robinson, Thermal explosions and the disappearance of criticality at small activation energies: exact results for the slab, Prov. Roy. Soc. London A., 368 (1979), pp. 441-468.

[3] D. W. FRADKIN AND G. C. WAKe, The critical explosion parameter of thermal ignition, J. Inst. Math. Appl., 20 (1977), pp. 471-484.

[4] J. P. Keener And H. B. Keller, Perturbed bifurcation theory, Arch. Rational Mech. Anal., 50 (1973), pp. 159-175.

[5] H. B. KELLER, Numerical solution of bifurcation and nonlinear eigenvalue problems, in Application of Bifurcation Theory, P. H. Rabinowitz, ed., Academic Press, New York, 1977, pp. 359-384.

[6] H. B. Keller AND R. K.-H. SzETO, Calculation of flows between rotating disks, Computing Methods in Applied Sciences and Engineering, Proc. 4th International Symposium, Versailles, 1979, NorthHolland, Amsterdam, 1980, pp. 51-61.

[7] G. MOORE AND A. SPENCE, The calculation of turning points of nonlinear equations, SIAM J. Numer. Anal., 17 (1980), pp. 567-576. 
[8] W. H. RAY, Bifurcation and stability problems in astrophysics, in Application of Bifurcation Theory, P. H. Rabinowitz, ed., Academic Press, New York, 1977, pp. 285-315.

[9] R. SEYDEL, Numerical computation of branches in nonlinear equations, Numer. Math., 33 (1979), pp. 339-352.

[10] A. SPEnCE AND B. Werner, Non-simple turning points and cusps, IMA J. Numer. Anal., 2 (1982) pp. 413-427.

[11] A. JePson AND A. SPEnCE, Folds in solutions of two parameter systems and their calculation: Part I., SIAM J. Numer. Anal., 22 (1985), pp. 347-368. 